\title{
Pseudogap Precursor of the Superconducting Gap in Under- and Overdoped $\mathrm{Bi}_{2} \mathrm{Sr}_{2} \mathrm{CaCu}_{2} \mathrm{O}_{8}+\delta$
}

\author{
Ch. Renner, ${ }^{1}$ B. Revaz, ${ }^{1}$ J.-Y. Genoud,${ }^{1}$ K. Kadowaki, ${ }^{2}$ and $\varnothing$. Fischer ${ }^{1}$ \\ ${ }^{1}$ DPMC, Université de Genève, 24, Quai Ernest-Ansermet, 1211 Genève 4, Switzerland \\ ${ }^{2}$ University of Tsukuba, Institute of Materials Science, Tsukuba, 305 Ibaraki, Japan
}

(Received 7 July 1997)

\begin{abstract}
We present tunneling spectroscopy of $\mathrm{Bi}_{2} \mathrm{Sr}_{2} \mathrm{CaCu}_{2} \mathrm{O}_{8+\delta}$ single crystals as a function of oxygen doping and temperature. The doping dependence amounts essentially to an energy scaling of the tunneling spectra, with a quasiparticle gap that is reduced with increasing oxygen concentration. This superconducting gap is temperature independent up to the superconducting transition where the superconducting spectra merge continuously into another gaplike feature at the Fermi level. This pseudogap is found to be present both in underdoped and overdoped samples, and it scales with the superconducting gap. [S0031-9007(97)04902-8]
\end{abstract}

PACS numbers: 74.50.+r, 74.25.Dw, 74.25.Jb, 74.72.Hs

The understanding of the origin and the nature of the pairing mechanism in high temperature superconductors (HTS's) represents a formidable challenge for both theory and experiment. One essential ingredient in the quest for possible mechanisms of this phenomenon is the quasiparticle density of states (DOS), whose detailed shape and temperature dependence are constraining parameters to any theoretical model. In addition to their unique superconducting properties, HTS's have a rich temperaturedoping $(T-\delta)$ phase diagram [1] which also has to be accounted for by theory. At low hole doping, these compounds are antiferromagnetic insulators. At large hole doping, they become typical metals. Superconductivity occurs in an intermediate region, and the doping with the highest superconducting transition temperature $\left(T_{c}\right)$ has been defined as optimal doping. Going from low to high hole doping, one expects a crossover from strong to weaker electron correlations; however, it is not known if the nature of the superconducting state and the gap function symmetry change as one goes from underdoped to overdoped superconductors.

Many experiments have provided evidence for unusual behavior in the normal state of underdoped HTS. Analysis of NMR [2], infrared conductivity [3], neutron scattering [4], transport properties [5], specific heat [6], thermoelectric power [7], spin susceptibility [8], and Raman spectroscopy [9] have uncovered evidence that below a certain temperature $T^{*}>T_{c}$ a gaplike structure is present in the electronic excitation spectra. More recently angular resolved photoemission studies $[10,11]$ have given direct evidence for the presence of a pseudogap in the DOS at the Fermi level $\left(E_{F}\right)$ above $T_{c}$. This gap was reported to exist only in underdoped samples and to disappear above a characteristic temperature $T^{*}$.

In this Letter we present direct measurements of the DOS by scanning tunneling spectroscopy on $\mathrm{Bi}_{2} \mathrm{Sr}_{2} \mathrm{CaCu}_{2} \mathrm{O}_{8+\delta}$ (Bi2212) single crystals. We show that the shape of the DOS in the superconducting state is essentially doping independent, that the pseudogap above
$T_{c}$ scales with the superconducting gap, and that it is also present in overdoped samples. Furthermore, both gaps are found to be essentially temperature independent.

Tunneling spectroscopy has been one of the fundamental tools in the study of the superconducting state [12]. This technique is particularly sensitive to the DOS near $E_{F}$, with the unique capability to probe the states above and below $E_{F}$. Tunneling spectroscopy is thus especially sensitive to any gap in the quasiparticle excitation spectrum at $E_{F}$ which shows up directly as a characteristic feature near zero bias in the raw data tunneling conductance curves. Compared to other techniques, tunneling spectroscopy has a very high energy resolution $\left(\sim k_{B} T\right)$ and appears as the ideal tool to investigate some of the questions raised here. Furthermore, using a scanning tunneling microscope (STM) to carry out such studies gives a very high spatial resolution $(\sim 0.1 \mathrm{~nm})$ so that possible inhomogeneities not seen by other techniques can be resolved. Thereby, STM can more easily separate intrinsic effects from extrinsic ones.

In our study we have explored part of the $T$ - $\delta$ phase diagram of $\mathrm{Bi}_{2} \mathrm{Sr}_{2} \mathrm{CaCu}_{2} \mathrm{O}_{8+\delta}$ single crystals for hole concentrations varying from slightly underdoped $\left(T_{c}=\right.$ $83.0 \mathrm{~K})$ to highly overdoped $\left(T_{c}=56.0 \mathrm{~K}\right)$. The superconducting transition temperature of each doping level defined as the superconducting transition onset using ac susceptibility measurements, are given in Fig. 1 together with the tunneling conductance to be discussed below. The transition widths lie in the range of 0.5 to $2.3 \mathrm{~K}$. Underdoped and overdoped samples were obtained by annealing the crystals in vacuum or in a high pressure oxygen atmosphere, respectively. The tunnel junctions $(R>0.9 \mathrm{G} \Omega$, defined at $+0.2 \mathrm{~V})$ were made between the cleaved Bi2212 (001) surface and a metallic tip of an STM [13]. We used Ir tips mounted perpendicular to the Bi-O layers. In this configuration, tunneling spectroscopy yields primarily an angular average over the $a b$-plane DOS of Bi2212. The energy scale of the tunneling spectra refers to the voltage applied to the sample. 
Thus, at negative sample bias, we are probing occupied electron states below $E_{F}$, similar to photoemission spectroscopy. We systematically checked our data for their reproducibility and significance following the procedure described in Ref. [14]. In particular, we verified that the tunneling spectra were spatially reproducible over $\mu \mathrm{m}$ sized regions (Fig. 1, inset) and that they did neither depend on time nor on tip-to-sample distance. Experimental details can be found in Refs. [13,14].

Like photoemission, tunneling spectroscopy is a surface sensitive probe and some concern arises as to whether bulk or surface related properties are measured. This point becomes crucial when investigating oxygen doping and temperature dependent features in the DOS. Aging effects have been reported in photoemission experiments [15] where the spectral line shape changed within a few hours. We did not observe any such effects. We measured the same surface for a period of up to four weeks, without any significant altering of the spectra. We also found that the tunneling spectra did not change after thermal cycling the sample through room temperature. Therefore, we believe that the results reported here reflect intrinsic oxygen doping and temperature dependencies of the DOS, and that they are not hampered by partial loss or absorption of oxygen in the topmost layers after cleaving.

We now focus on the oxygen doping dependence of the tunneling spectra on Bi2212 at $4.2 \mathrm{~K}$, shown in Fig. 1. The striking result is that underdoped and overdoped samples have the same generic tunneling density of states as optimally doped Bi2212 [14]. In the gap region, the tunneling spectra show well developed peaks at the gap edges $\left( \pm \Delta_{p}\right)$, a finite conductance below the gap, and

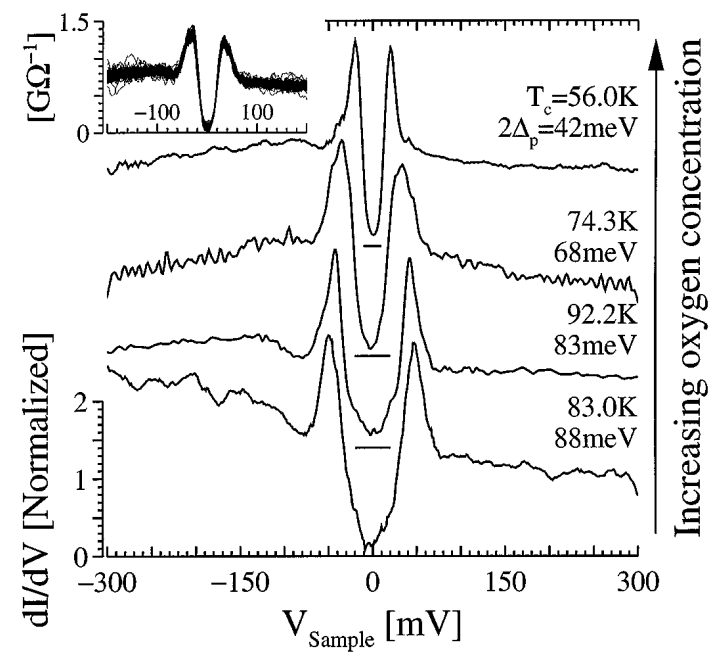

FIG. 1. Tunneling spectra measured at $4.2 \mathrm{~K}$ for different oxygen doping levels. The curves are normalized to the conductance at $200 \mathrm{mV}$ and offset vertically for clarity (zero conductance is indicated for each spectrum by the horizontal line at zero bias). The estimated error on the gap values $\left(2 \Delta_{p}\right)$ is $\pm 4 \mathrm{meV}$. The inset shows 200 superposed spectra measured at equally spaced points along a $0.15 \mu \mathrm{m}$ line on overdoped Bi2212 $\left(T_{c}=71.4 \mathrm{~K}\right)$, demonstrating the spatial reproducibility. a dip at approximately $-2 \Delta_{p}$ which appears at negative bias only as reported earlier [14]. The major effect of increasing oxygen doping is to shift the conductance peaks at $\pm \Delta_{p}$ and the dip near $-2 \Delta_{p}$ toward $E_{F}$. The energy dependence of the conductance at low bias is not consistent with a fully gapped Fermi surface expected for a conventional BCS superconductor, but corresponds very well to the expected shape for a $d$-wave pair state [16] if some impurity scattering is taken into account [17]. The finite conductance below the gap is thus consistent with the presence of nodes in the gap function (see also the fits in Ref. [14]). Since the energy dependence of the conductivity at low bias is very sensitive to any changes in the pairing symmetry through the node structure, the fact that the conductivity below the gap is basically not affected by doping, neither in its magnitude nor in its energy dependence, is remarkable. It is a strong indication that the symmetry of the pairing interaction is not fundamentally modified upon doping. This result is in contradiction to some photoemission studies which found changes in the gap anisotropy with oxygen doping [18].

The magnitude of the superconducting gap can in principle be derived directly from the tunneling spectra. However, in the absence of an established model for the gap function and the DOS in HTS's, such a quantitative analysis is not straightforward. Thus, in order to compare different spectra, we assign the gap amplitude $\Delta_{p}$ in a more phenomenological way as half the spacing between the conductance peaks at $\pm \Delta_{p}$. This definition corresponds to the maximum gap in a $d$-wave or extended $s$-wave pairing symmetry. The values for $2 \Delta_{p}$ are listed in Fig. 1, which illustrates the reduction of the superconducting gap with increasing oxygen concentration. The reduced gap $2 \Delta_{p} / k_{B} T_{c}$ equals $12.3 \pm 1$ in the underdoped single crystals and gradually diminishes to $8.7 \pm 1$ in the overdoped ones. It is remarkable that the very high values of $2 \Delta_{p} / k_{B} T_{c}$ persist up to the highest doping level and only a slight decrease is found compared to optimal doping. There is no sign that the superconducting state becomes more BCS-like in the highly overdoped samples. The doping dependence of the gap we find [19] is in contradiction to earlier tunneling experiments where the gap was found to be independent of oxygen concentration [20], although other experiments agree with the present findings [21]. So far, we measured the largest gap amplitude in the $83 \mathrm{~K}$ underdoped Bi2212 samples, indicating that the gap is increasing in the underdoped samples although $T_{c}$ is decreasing, as suggested in recent experiments [21,22].

We now turn to the temperature dependence of the quasiparticle DOS in Bi2212. Figure 2 shows a set of tunneling conductance curves taken at temperatures between 4.2 and $293 \mathrm{~K}$ on an underdoped sample with $T_{c}=83 \mathrm{~K}$. The temperature dependence is very unusual compared to conventional BCS superconductors, and one sees immediately some striking features of these spectra. All curves show a gaplike structure with an energy scale that to first approximation does not change with temperature. The 
superconducting gap remains temperature independent up to $T_{c}$ where no sign is indicating that it is closing. Across $T_{c}$, the superconducting tunneling spectra evolve continuously into a normal state quasiparticle gap structure, which we refer to as a pseudogap. It is clear from Fig. 2 that this pseudogap also does not change much with temperature and that it is even possibly there at room temperature. The pseudogap structure just gets weaker with increasing temperature. This is in striking contrast to photoemission data which for a sample with the same doping level led to the conclusion that the gap reduced to zero at $T^{*}=170 \mathrm{~K}$ [11]. We believe the $T^{*}$ observed in various experiments is to be considered as a characteristic energy scale and not as a temperature where the pseudogap is reduced to zero. At first sight it is not clear from these data where the critical temperature is located. A closer inspection of the spectra shows that the peak at negative energy and the dip at about $-2 \Delta_{p}$ disappear at $T_{c}$. The positive bias peak on the measured spectra does not vanish at $T_{c}$, but remains finite and shifts to higher energies above $T_{c}$ [Fig. 3(b)]. The DOS at the Fermi level bears no particular signature of the superconducting transition in this set of data. Indeed, the DOS below the gap starts to be depleted already at room temperature and gradually hollows in to reach its minimum at $4.2 \mathrm{~K}$. This low bias temperature dependence is better seen in Fig. 3(a), which is a three dimensional plot of the tunneling conductance as a function of energy and temperature of the $83 \mathrm{~K}$ underdoped $\mathrm{Bi} 2212$. The same data are displayed as a gray scale projection onto the energy-temperature plane in Fig. 3(b), where white corresponds to high conductivity $\left(1.5 \mathrm{G} \Omega^{-1}\right)$ and black to zero conductivity.

So far most measurements report the existence of a pseudogap in underdoped samples. Our measurements

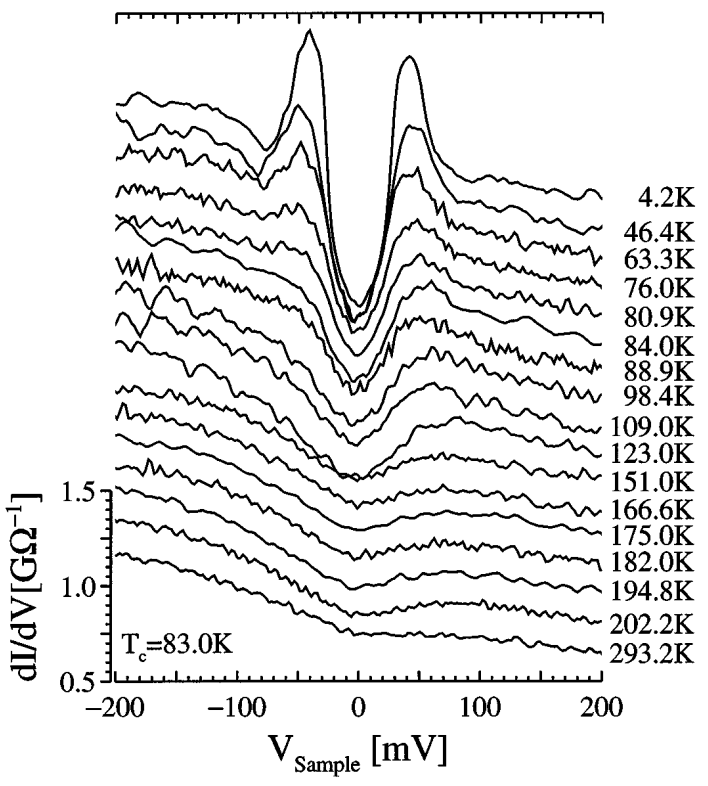

FIG. 2. Tunneling spectra measured as a function of temperature on underdoped Bi2212. The conductance scale corresponds to the $293 \mathrm{~K}$ spectrum, the other spectra are offset vertically for clarity. show that the pseudogap is present above $T_{c}$ both at optimum doping and in overdoped samples. In Fig. 4 we show a set of spectra for the overdoped sample with a $T_{c}$ of $74.3 \mathrm{~K}$. We see that the behavior is precisely the same as for the underdoped case discussed above. The gap value stays temperature independent and the peak at negative energy as well as the dip at about $-2 \Delta_{p}$ disappear at $T_{c}$, but the region below the gap evolves smoothly across $T_{c}$ into the pseudogap. The difference with the underdoped case is that the gap and pseudogap are smaller, and the amplitude of the pseudogap structure seems to disappear more quickly as the temperature is increased. The fact that the pseudogap scales with the superconducting gap and that it is smaller in the overdoped samples than in the underdoped ones indeed demonstrates that the pseudogap in Fig. 4 is a property of the overdoped crystal, and not that of an underdoped surface resulting from partial loss of oxygen at high temperature. The two curves shown on top of the $69 \mathrm{~K}$ spectrum in Fig. 4 illustrate the temperature independence of the gap. Simply thermally smearing the $4.2 \mathrm{~K}$ spectrum to $69 \mathrm{~K}$ (dashed curve, $\Delta_{69 \mathrm{~K}}=\Delta_{4.2 \mathrm{~K}}$ ) reproduces much better the position of the conductance peaks at $69 \mathrm{~K}$ than thermally smearing the $4.2 \mathrm{~K}$ spectrum to $69 \mathrm{~K}$ assuming a reduced gap (dotted curve, $\Delta_{69 \mathrm{~K}}=$ $0.8 \Delta_{4.2 \mathrm{~K}}$ ). The numerical spectra show that there are more low energy states measured than predicted by simply thermally smearing the data. However, this feature is beyond the scope of this Letter. We have also carried out similar analysis for the $83 \mathrm{~K}$ underdoped sample with basically the same conclusions.

Several theoretical studies have considered the possibility of the presence of superconducting phase fluctuations

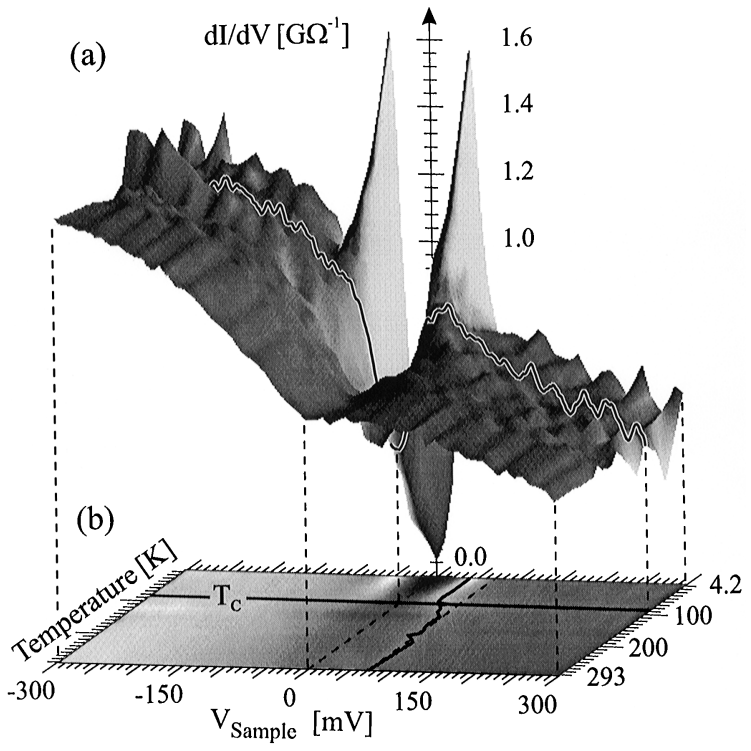

FIG. 3. (a) Three dimensional view of the conductance data shown in Fig. 2. The highlighted curve is the spectrum measured at $T_{c}$. (b) Projection onto the energy-temperature plane. The line at positive bias indicates the position of the positive bias conductance peak which clearly shifts to higher energies above $T_{c}$. 
[23] or spin fluctuations [24] above $T_{c}$. Either of these ideas may in principle explain the existence of a pseudogap. However, our results put some constraints on such theories. First, in the samples we have investigated we find that the energy scale of the superconducting gap and the pseudogap is the same in a given sample, or in other words, the amplitude of the pseudogap scales with the superconducting gap. Furthermore, the pseudogap is centered at the Fermi level in both under- and overdoped samples. It is therefore unlikely that the pseudogap results from a band structure effect. We believe that our results contain new evidence that the pseudogap is directly related to superconductivity and shows either the presence of important superconducting fluctuations or preformed pairs above $T_{c}$. It is remarkable that there is no sign that the pseudogap closes at a given temperature. If this happens, it must happen above room temperature in the $83 \mathrm{~K}$ underdoped sample and above $89 \mathrm{~K}$ in the $74.3 \mathrm{~K}$ overdoped sample. The detailed analysis presented in Figs. 2 and 3 reveals that the measured density of states above $T_{c}$ is asymmetric in that there is clearly a peak at positive energies, whereas at negative energies the peak disappears at $T_{c}$. We believe that this is a characteristic signature of the pseudogap state.

In summary, we find that the superconducting quasiparticle density of states in both under- and overdoped $\mathrm{Bi}_{2} \mathrm{Sr}_{2} \mathrm{CaCu}_{2} \mathrm{O}_{8+\delta}$ has essentially the same shape as for the optimally doped case. The effect of increasing doping amounts essentially only to reducing the energy scale of the tunneling conductance curves. We believe this demonstrates that the nature and the symmetry of the su-

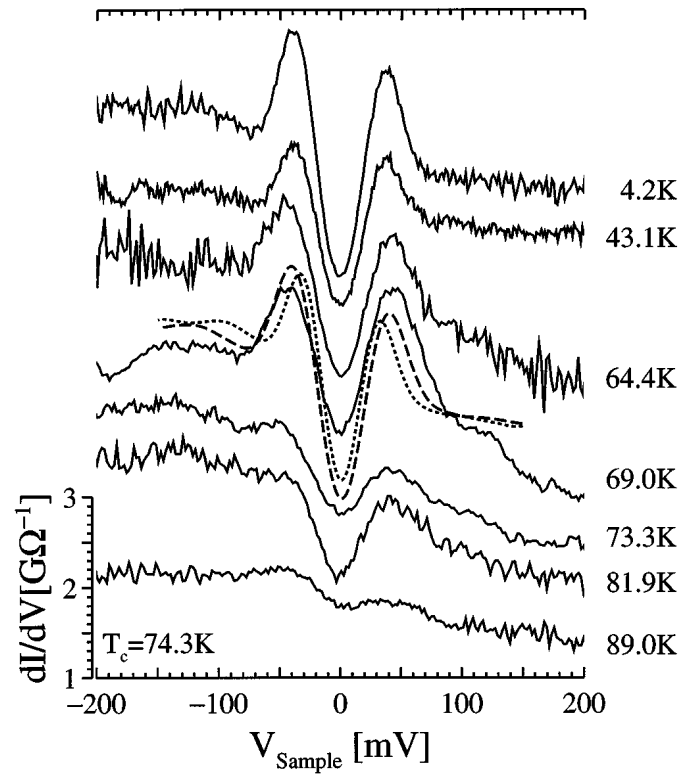

FIG. 4. Tunneling spectra measured as a function of temperature on overdoped $\mathrm{Bi} 2212$. The conductance scale corresponds to the $89 \mathrm{~K}$ spectrum, the other spectra are offset vertically for clarity. The dashed spectrum corresponds to the $4.2 \mathrm{~K}$ spectrum thermally smeared to $69 \mathrm{~K}\left(\Delta_{69 \mathrm{~K}}=\Delta_{4.2 \mathrm{~K}}\right)$. The dotted spectrum is obtained the same way, but assuming a reduced gap $\left(\Delta_{69 \mathrm{~K}}=0.8 \Delta_{4.2 \mathrm{~K}}\right)$. perconducting state is doping independent. A striking result is that the gap in the underdoped region continues to increase although $T_{c}$ decreases. The evolution with temperature is very different from the classic BCS behavior. The superconducting gap is temperature independent and does not at all close at $T_{c}$. Instead the tunneling spectra evolve continuously into a new structure reflecting a pseudogap with essentially the same magnitude. This is observed both for underdoped samples with a large gap and for overdoped samples where both gaps are smaller, strongly suggesting that the pseudogap has the same origin as the superconducting gap.

We thank C. Ahn for his careful reading of the manuscript. We are grateful to J.-G. Bosch for his technical assistance. This work was supported by the Swiss National Science Foundation.

[1] See, for instance, B. Batlogg, Phys. Today 44, No. 6, 44 (1991).

[2] H. Alloul, T. Ohno, and P. Mendels, Bull. Am. Phys. Soc. 34, 633 (1989); Phys. Rev. Lett. 63, 1700 (1989); W. W. Warren et al., Phys. Rev. Lett. 62, 1193 (1989); G. V. M. Williams et al., Phys. Rev. Lett. 78, 721 (1997).

[3] S. L. Cooper et al., Phys. Rev. B 40, 11358 (1989); L. D. Rotter et al., Phys. Rev. Lett. 67, 2741 (1991); A. V. Puchkov et al., Phys. Rev. Lett. 77, 3212 (1996).

[4] J. Rossat-Mignod et al., Physica (Amsterdam) 169B, 58 (1991).

[5] H. Y. Hwang et al., Phys. Rev. Lett. 72, 2636 (1994); B. Batlogg et al., Physica (Amsterdam) 235C, 130 (1994).

[6] J. W. Loram et al., Phys. Rev. Lett. 71, 1740 (1993).

[7] J. L. Tallon et al., Phys. Rev. Lett. 75, 4114 (1995).

[8] W. Y. Liang et al., Physica (Amsterdam) 263C, 277 (1996).

[9] R. Nemetschek et al., Phys. Rev. Lett. 78, 4837 (1997).

[10] A. G. Loeser et al., Science 273, 325 (1996).

[11] H. Ding et al., Nature (London) 382, 51 (1996).

[12] E. L. Wolf, Principles of Electron Tunneling Spectroscopy (Oxford University Press, Oxford, 1989).

[13] Ch. Renner et al., J. Vac. Sci. Technol. A 8, 330 (1990); A. D. Kent et al., Ultramicroscopy 42-44, 1631 (1992).

[14] Ch. Renner and Ø. Fischer, Phys. Rev. B 51, 9208 (1995).

[15] H. Ding et al., Phys. Rev. Lett. 78, 2628 (1997).

[16] D. J. Scalapino, Phys. Rep. 250, 329 (1995); H. Won and K. Maki, Phys. Rev. B 49, 1397 (1994).

[17] T. Hotta, J. Phys. Soc. Jpn. 62, 274 (1993).

[18] R. J. Kelley et al., Science 271, 1255 (1996).

[19] Ch. Renner et al., J. Low Temp. Phys. 105, 1083 (1996).

[20] J. Liu, Y. Li, and C. M. Lieber, Phys. Rev. B 49, 6234 (1994).

[21] M. Oda et al., Physica (Amsterdam) 282-287C, 1499 (1997).

[22] J. M. Harris et al., Phys. Rev. B 54, (1996).

[23] V.J. Emery and S. A. Kivelson, Nature (London) 374, 434 (1995); M. Randeria et al., Phys. Rev. Lett. 69, 2001 (1992).

[24] T. Tanamoto, K. Kohno, and H. Fukuyama, J. Phys. Soc. Jpn. 61, 1886 (1992); J. R. Schrieffer and A.P. Kampf, J. Phys. Chem. Solids 56, 1673 (1995); D. Pines, Z. Phys. B 103, 129 (1997). 